\title{
Lumen
}

Selected Proceedings from the Canadian Society for Eighteenth-Century Studies

\section{La " Nature feminine " ou la fin du libertinage : Les Amours du Chevalier de Faublas de Louvet (1787-1790)}

\section{Catherine Cusset}

Volume 12, 1993

URI : https://id.erudit.org/iderudit/1012585ar

DOI : https://doi.org/10.7202/1012585ar

Aller au sommaire du numéro

Éditeur(s)

Canadian Society for Eighteenth-Century Studies / Société canadienne d'étude du dix-huitième siècle

ISSN

1209-3696 (imprimé)

1927-8284 (numérique)

Découvrir la revue

Citer cet article

Cusset, C. (1993). La « Nature feminine » ou la fin du libertinage : Les Amours du Chevalier de Faublas de Louvet (1787-1790). Lumen, 12, 131-137.

https://doi.org/10.7202/1012585ar

Copyright (c) Canadian Society for Eighteenth-Century Studies / Sociéte canadienne d'étude du dix-huitième siècle, 1993
Ce document est protégé par la loi sur le droit d'auteur. L'utilisation des services d'Érudit (y compris la reproduction) est assujettie à sa politique d'utilisation que vous pouvez consulter en ligne.

https://apropos.erudit.org/fr/usagers/politique-dutilisation/ 


\section{La 'Nature feminine' ou la fin du libertinage: Les Amours du Chevalier de Faublas de Louvet (1787-1790)}

On aimerait croire au bonheur dans le libertinage tel que l'exposent les tableaux de Watteau, de Boucher et de Fragonard: pèlerinages à l'île de Cythère, nudités joyeuses, baisers volés dans l'innocence. Mais la fiction romanesque, dès le milieu du dix-huitième siècle, le dément: les derniers romans de Crébillon-fils, Les Confessions $d u$ Comte de ${ }^{* * *}$ de Pinot-Duclos, Les Malheurs de l'Inconstance de Dorat, Les Liaisons Dangereuses de Laclos, semblent nier la possibilité d'un libertinage heureux conciliant l'amour et les plaisirs sans la nécessité d'un choix. Par là-même, ils mettent en question l'innocence de la nature et la réalité du bonheur 'organique' ou physiologique qui a suscité tant de polémiques au cours du XVIIIème siècle.

Une exception: le roman de Louvet de Couvrai, Les Amours $d u$ Chevalier de Faublas, publié entre 1787 et 1790, représente un absolu d'innocence dans le libertinage. Le lecteur sera frappé par l'absence d'une intrigue centrale donnant un axe au roman, allant de pair avec l'innocence du héros narrateur. Faublas ne manigance pas une seule 'liaison dangereuse,' mais s'abandonne au hasard de ses coups de foudre, à sa nature dont l'instinct ne peut être contredit, puisqu'elle est son être même, la manière dont il est 'fait': 'Que voulez-vous! Je suis ainsi fait: dans aucune circonstance de ma vie, quelque embarrassante que vous l'imaginiez, je n'ai pu voir de près plusieurs femmes ensemble sans avoir de longues distractions' (757). ${ }^{1}$ A l'inconstance de Faublas, il n'y a rien à répondre. Faublas, et derrière lui Louvet, à la manière d'un philosophe matérialiste et polémique comme La Mettrie, ne cesse de s'adresser au lecteur 'censeur': il est inutile de moraliser, car la morale est impuissante face à la nature, et la nature, qui n'est autre que le désir ou même plus simplement la puissance ou l'impuissance sexuelle, suffit à se régir elle-même.

Une telle innocence, une telle confiance en la nature sont-elles possibles? Louvet l'affirme pendant huit-cents pages, avant d'achever subitement et dramatiquement son immense roman avec la mort des deux maîtresses de Faublas et la folie passagère de son héros: les 
mémoires du libertin heureux se terminent par un échange épistolaire de vingt pages où le héros annonce sa 'guérison,' suivant en cela, semble-t-il, le schéma traditionnel du roman libertin. Pourquoi cette fin? Qu'en est-il de l'innocence du héros, sans cesse réaffirmée durant les nombreuses pages de la narration? Louvet veut-il finalement nous dire, lui aussi, l'impossibilité d'un libertinage heureux en harmonie avec la nature?

Michel Delon a consacré aux Amours du Chevalier de Faublas un article intitulé: 'Rupture et transition dans le roman libertin à la fin de l'Ancien Régime, ${ }^{2}$ dans lequel il insiste sur le rôle joué par un personnage du roman, le baron du Portail alias Lovzinski, un Polonais émigré, ami du père de Faublas. Le récit de ses héroïques aventures est inséré dans le roman: il avait dû quitter son pays envahi par les Russes, et avait perdu sa fille, enlevée par eux au cours d'un combat. A la fin de la première partie du roman, 'Une Année de la Vie de Faublas' (publié en 1787), Lovzinski/du Portail retrouve sa fille perdue en la personne de Sophie, une des amantes de Faublas, déjà séduite par lui. Son amitié pour Faublas et sa complicité protectrice se transforment alors en rage vengeresse: il marie les deux amants pour réparer l'honneur perdu, puis il enlève sa fille, en avertissant Faublas qu'il la lui rendra seulement lorsqu'il sera digne d'elle, c'est-à-dire lorsqu'il aura retrouvé le sens des responsabilités conjugales, sociales et historiques.

Michel Delon écrit: 'la fiction de Louvet est dominée par le contraste entre le tourbillon des aventures galantes à Paris et l'héroïsme d'aventures d'un tout autre registre en Pologne' (109). En accordant une place centrale à la voix narrative de Lovzinski, le critique réinstalle la dichotomie que le roman semblait éliminer par sa pluralité $d$ 'intrigues. Une fois la dichotomie rétablie, il conclut au retour d'un système de valeurs à l'intérieur du roman. Le personnage de Lovzinski servirait ainsi à redonner à la 'nature' les limites sociales, morales et politiques sans lesquelles elle n'aurait pas de sens, mettant par là fin au libertinage heureux et irresponsable.

Cette interprétation se justifierait si l'enlèvement de Sophie par son père transformait en effet la mascarade des intrigues libertines en une histoire simple conduisant le roman vers sa fin logique, les retrouvailles du héros repenti avec sa femme, et contraignait ainsi le héros à une quête de l'unique. C'est peut-être ce que souhaiterait le lecteur: que le héros suive logiquement son désir et se moralise. Mais ce n'est pas ce qui se produit: l'acte du père a peu de conséquences sur l'intrigue même du roman, qui reste légère et libertine. Son désir de retrouver Sophie n'empêche pas Faublas de s'arrêter en cours de route pour satisfaire d'autres désirs. Plus le roman progresse, et plus l'intrigue se disperse; plus le nombre de femmes arrêtant Faublas dans sa quête se multiplie, 
scandalisant le père de Faublas: 'Quoi! les infortunes de Sophie et son sort incertain ne peuvent-ils vous occuper assez? Faut-il absolument que plusieurs objets exercent à la fois l'activité de votre âme et l'inconstance de vos sens?' (869). Au lieu de se resserrer et de se finaliser, l'intrigue se défait au fur et à mesure que le roman progresse et que le chevalier se rapproche de son but apparent. L'ironie du roman consiste dans un refus de satisfaire l'attente du lecteur, attente construite par le roman luimême: 'Je partis, je partis te rejoindre, ma Sophie; mais combien de malheurs, que d'ennemis et de rivales devaient encore retarder le moment de notre réunion!'(754). Le roman se joue de l'intrigue qu'il a feint de mettre en place.

Loin de déterminer le héros à se rendre digne de sa femme, l'enlèvement de Sophie par son père le livre à l'indétermination: 'Si je n'enlève mon Eléonore, elle est perdue; ma Sophie, si je l'abandonne, meurt de chagrin. Quel homme à ma place ... pourrait encore se déterminer?' (1197). L'éducation morale tentée par Lovzinski est un échec. Même au moment le plus dramatique du roman, entre le meurtre de Mme de $B^{* * *}$ et le suicide de Mme de Lignolle, ses deux maîtresses, alors que la mort semble rendre impossible l'innocence du libertinage heureux, Faublas persiste à refuser toute culpabilité: 'Des remords, grands dieux! et pourquoi? Vous m'avez donné le coeur le plus aimant et les sens les plus vifs; vous avez voulu que je rencontrasse à la fois plusieurs femmes, exprès ornées pour plaire aux yeux et charmer l'âme...'(1196). Le 'coeur' et les 'sens,' définissant la nature de Faublas, servent jusqu'à la fin à nier le fondé d'une morale réprimant la nature.

Se pose alors la question suivante: pourquoi la chaîne d'intrigues, d'inconstances et de plaisirs s'achève-t-elle avec la mort des deux maîtresses, le meurtre de Mme de $B^{* * *}$ par son mari et le suicide de Mme de Lignolle provoquant la folie passagère de Faublas? Qu'est-ce qui détermine la fin dramatique du roman?

Rien, au cours du récit (pourtant rétrospectif), ne dirige le roman vers sa fin: rien, sinon sa fin, ne permet au roman de finir. Une telle proposition semble tautologique; elle ne l'est pas: j'oppose ici le roman de Louvet aux autres romans libertins ou à un roman comme Manon Lescaut où le regard rétrospectif du narrateur fait présager dès le début le dénouement dramatique et la reconnaissance par le héros de ses 'égarements.' Dans Les Amours de Faublas, rien, jusqu'aux trente dernières pages, ne permet d'anticiper la fin. Cette fin, c'est la mort volontaire des deux maîtresses de Faublas, Mme de $B^{* * *}$ et Mme de Lignolle, qui supprime la possibilité du pluriel des 'amours' de Faublas, en ne laissant sur scène que la femme légitime, Sophie. Si, donc, morale il y a, c'est du côté des femmes que Louvet nous invite à la chercher. 
On attendrait des héroïnes aussi légères et heureuses que le libertin qu'elles chérissent. Les femmes du roman, alors même qu'elles sont les victimes de l'inconstance de Faublas, reconnaissent paradoxalement leur faute: 'Il est donc vrai, me dit-elle en sanglotant, qu'une première faute entraîne toujours une faute plus grave!'(714). Les termes appartenant à un registre de vocabulaire moral, 'faute, 'égarements,' 'fin tragique,' 'trahison,' ne se trouvent que dans la bouche des personnages féminins du roman, comme si les femmes incarnaient une moralité dérisoire dans un roman qui en est par ailleurs dépourvu.

De quelle moralité s'agit-il? Les femmes séduites et infidèles à leur mari se repentent-elles un peu tard d'avoir cédé à leur désir? L'infidélité conjugale n'est pas la faute qui trouble leur conscience: elles la revendiquent comme un droit. 'Faublas, vous connaissez le marquis; on m'a mariée malgré moi, on m'a sacrifiée' (512), dit la marquise de $\mathrm{B}^{* * *}$, et la comtesse de Lignolle justifie de la même manière sa liaison adultère avec Faublas. On reconnaît ici un discours souvent attribué aux femmes des romans libertins au XVIIIème siècle, revendiquant l'infidélité comme le seul moyen de reconquérir une liberté dont les a privées la société.

De quelle faute s'agit-il alors? Si l'on analyse les scènes au cours desquelles les femmes du roman prononcent le mot 'faute,' on s'aperçoit qu'il n'est employé par Louvet que dans certains contextes précis: lorsque les héroïnes ont la preuve de l'infidélité de Faublas. C'est alors que leur amour se transforme en 'faute.' Dans cette 'faute,' il faut plutôt voir un défaut, un manque, qu'une faute au sens moral: l'amour des femmes ne devient 'faute' qu'à partir du moment où le héros leur fait défaut. L'aveu de culpabilité des femmes du roman est la reconnaissance d'un manque.

En dépit de leurs différences, toutes les femmes des Amours $d u$ Chevalier de Faublas expriment en effet le même désir: le désir de s'attacher Faublas. Le roman révèle progressivement que ce désir est le seul qui donne un sens à leurs actions. 'Je vous déclare que je pars avec vous pour Compiègne, que je vous suis partout, que je m'attache à vous comme votre ombre' (922), dit la comtesse de Lignolle, craignant d'être abandonnée de Faublas. Elle rêve d'une petite maison où elle pourra se réfugier avec son amant, d'une cabane où il n'y aura qu'un lit et qu'une assiette: 'Qu'il y ait seulement de quoi loger une personne, car nous ne serons qu'un' (1110). Mais Faublas, amoureux de la comtesse de Lignolle, reste en même temps le tendre époux de Sophie et l'admirateur de la marquise de $B^{* * *}$. Il demeure insaisissable, et c'est cette impossibilité de le 'fixer' qui provoque le désespoir de la comtesse de Lignolle, transformant son amour en 'faute.' Qu'une femme aussi jeune que Mme de Lignolle désire s'attacher à jamais son premier 
amant ne peut guère étonner le lecteur, de même qu'on ne peut être surpris de voir Sophie aspirer à la fidélité de son époux. Beaucoup plus surprenante est la confession finale de la marquise de $B^{* * *}$.

Louvet semble en effet avoir emprunté à Laclos la tripartition des caractères féminins établie par Mme de Merteuil dans sa grande lettre autobiographique: elle distingue les 'femmes à délire' ou 'à sentiments,' les 'femmes sensibles,' et les femmes de tête, qui, comme Mme de Merteuil elle-même, suivent seulement les principes qu'elles se sont créés (Les Liaisons Dangereuses, lettre 81). Sophie, femme légitime de Faublas, serait la femme sensible, Mme de Lignolle, folle de Faublas, la femme à délire, et enfin, Mme de $B^{* * *}$, seule capable de remplacer le sentiment immédiat par la pensée et les principes, la femme de tête. On apprend en effet à la fin du roman que $\mathrm{Mme}_{\mathrm{de}} \mathrm{B}^{* * *}$ avait elle-même conçu la plupart des intrigues et des aventures auxquelles a participé Faublas.

Et pourtant, Mme de $B^{* * *}$ finit elle aussi par reconnaître sa faute: 'J'osai te revoir, je l'osai! Ce fut ma première faute, elle prépara mes derniers égarements et ma fin tragique' (1146). Lors de sa confession finale, juste avant de mourir, Mme de $B^{* * *}$ trahit son secret: elle révèle que ses ruses et ses stratégies ont été déterminées non par le goût du jeu et du plaisir qui entraîne Faublas, mais par le désir de garder Faublas pour elle seule: 'Tu verras si je suis femme à partager un amant!'(1189).

Il est essentiel de comprendre que cette conception de l'amour partagée par les trois héroïnes du roman ne repose pas sur un fondement moral, et ne contredit pas, en ce sens, l'absence de moralité du roman: si la femme est fidèle à l'homme aimé, constante dans sa passion, et exclusive, ce n'est ni par devoir moral, ni par devoir social, mais par une nécessité ontologique: 'Mais comment fait-il donc pour aimer ainsi tout le monde! Moi, je ne puis aimer qu'un homme! Je ne puis idolâtrer que lui!' (970), s'écrie la comtesse de Lignolle en dénonçant sa propre constance amoureuse comme une impuissance de nature.

La reconnaissance, par toutes les femmes des Amours du Chevalier de Faublas, de leur désir d'exclusivité amoureuse, conduit à la conclusion suivante: tout comme Faublas, les femmes obéissent à leur nature; mais la nature féminine se définit par un désir irrésistible de fixation de l'autre. Ce n'est pas le libertinage de Faublas, mais le désir féminin de fixation qui est à l'origine de la fin tragique du roman. Comme la voix narrative appartient au personnage libertin incapable de concevoir une telle forme de désir, le roman reste léger et joyeux en dépit de sa fin: Faublas est simplement la victime du désir des femmes, elles-mêmes victimes de leur propre désir. 
La manière dont Louvet montre l'impossibilité d'un libertinage heureux diffère fondamentalement de celle de ses contemporains: afin d'achever son roman sans contredire l'innocence de son héros, il définit le féminin comme l'impossibilité ontologique du libertinage. En ce sens, ce roman marque la fin du XVIIlème siècle. Il est la négation même des Liaisons Dangereuses: si Mme de Tourvel se meurt d'amour, la fuite de Mme de Merteuil en Hollande avec tous ses bijoux est plus ambiguë et ne permet pas de conclure à la défaite de la femme galante. Pour Louvet, Mme de Merteuil, le type des femmes de tête, n'existe pas: il n'y a que des Mme de $B^{* * *}$, que des femmes en qui triomphe la 'nature,' définie par Louvet, du côté féminin, comme l'amour exclusif pour un seul homme. ${ }^{3}$

Les Amours du Chevalier de Faublas, publié pendant la révolution française, appartient en même temps à deux mondes. Le libertinage de Faublas n'est jamais condamné: en cela, le roman de Louvet est un roman d'ancien régime, et la versatilité amoureuse du héros le signe de son irresponsabilité politique. Néanmoins, pour que le roman s'achève, Louvet confronte son héros avec une réalité qui n'est pas d'ordre politique ou moral, mais psychologique: cette réalité, c'est celle du désir féminin, un désir de constance et de fixation qui provoque la fin du pluriel des amours. Par sa fin, le roman de Louvet appartient au nouveau monde, celui de la révolution et de la république qui ouvre sur le XIXème siècle: la deuxième et la troisième partie du roman, publiées en 1789 et 1790, se situent au coeur de la fracture révolutionnaire, qui substitue au mythe de l'ancien régime, celui d'une femme sexuellement dévorante - incarnée par Marie-Antoinette - ${ }^{4}$ une nouvelle femme sans autre désir que celui de sa maison et de sa famille, Marianne vertueuse et républicaine. ${ }^{5}$ On ne sera pas surpris d'apprendre que Louvet, le 10 février 1792, a fait décider l'exclusion des femmes des débats des Jacobins. ${ }^{6}$ Par son dénouement, le roman de Louvet, Les Amours du Chevalier de Faublas, nous dit qu'en toute Mme de Merteuil se cache une Mme de Tourvel, et que les actes d'une femme recèlent une motivation unique: l'amour fidèle à un seul homme, amour qui est le fondement de la famille et de la vertu. Ainsi s'achève le roman-hydre des amours libertines: décapité par une fin bourgeoise. ${ }^{7}$

\section{CATHERINE CUSSET}

Yale University 


\section{Notes}

1 Louvet de Couvrai, Les Amours du Chevalier de Faublas, dans Romanciers du XVIIIème siècle, tome II (Paris: Gallimard, Bibliothèque de la Pléiade, 1965).

2 Voir Michel Delon, 'Rupture et transition dans le roman libertin à la fin de l'ancien régime: Louvet et Nerciat,' Signes du Roman, Signes de la Transition (Paris: PUF, 1986).

3 La critique littéraire a souvent tenté d'expliquer psychologiquement le personnage de Mme de Merteuil, en concluant qu'elle était 'en vérité,' derrière tous ses masques, amoureuse du vicomte de Valmont. Il est significatif que le beau film de Stephen Frears, prototype de cette interprétation psychologique, nous montre une Mme de Merteuil versant des larmes sur son désir incompris, alors que Laclos n'indique jamais qu'une seule larme ait été versée par son héroïne. Le roman permet au lecteur de supposer, s'il le désire, une telle motivation psychologique, mais il ne l'établit certainement pas comme une vérité, restant profondément ambigu. Voir Henri Duranton, 'Les Liaisons Dangereuses ou le miroir ennemi,' Revue des Sciences Humaines 153 (1974): 125-143, et Peter Brooks, The Novel of Worldliness (Princeton N.J.: Princeton UP, 1969). Pour une analyse des quatre versions cinématographiques des Liaisons Dangereuses (celles de Roger Vadim en 1959, de Brabant en 1979, de Frears en 1988, et de Forman en 1989), voir Alan J. Singerman, 'Variations on a Denouement: Les Liaisons Dangereuses on Film,' Eighteenth Century Life 14.2 (1990): 49-55.

4 Voir Chantal Thomas, La Reine Scélérate: Marie-Antoinette dans les Pamphlets (Paris: Seuil, 1989); voir aussi Lynn Hunt, 'The Many Bodies of Marie-Antoinette:

Political Pornography and the Problem of the Feminine in the French Revolution,' Eroticism and the Body Politic, ed. Lynn Hunt (Baltimore: Johns Hopkins UP, 1991) 108-30.

5 Voir Philippe Ariès et George Duby, gen. ed., Histoire de la Vie privée, vol. 4 (Paris: Seuil, 1987), et Georges Duby et Michelle Perrot, gén. éd., Histoire des Femmes, vol. 3 (Paris: Plon, 1991).

6 Voir Michel Vovelle, 'La vérité dans la fantasme,' préface aux Mémoires de Jean-Baptiste Louvet (Paris: Editions Desjonquères, 1988) IX.

7 'Moi, je suis père; il n'y a pas tout-à-fait quatre mois que Sophie m'a donné le plus joli garçon du monde. Ma Sophie ... devient chaque jour plus adorable,' annonce Faublas dans sa dernière lettre (1222). 